\title{
Carcinoma adenoideo quístico de mama: serie de casos
}

\author{
Sheila Huertas T.a, Elizabeth Zegarra B. ${ }^{a}$, Domingo Morales ${ }^{1, b}$, Valentín Jaimes \\ Serkovic. ${ }^{2, b}$ \\ ${ }^{1}$ Servicio de Patología, ${ }^{2}$ Servicio de Ginecología Oncológica, Hospital Nacional Edgardo Rebagliati Martins. Lima, Perú. \\ a Estudiante de Medicina Humana, UPC (Universidad Peruana de Ciencias Aplicadas). ${ }^{\mathrm{b}}$ Docente de UPC (Universidad \\ Peruana de Ciencias Aplicadas). Lima, Perú.
}

\section{RESUMEN}

El carcinoma adenoideo quístico (CAQ) de mama es un cáncer poco frecuente y representa entre el 0,1\% y el $0,4 \%$ de todos los carcinomas de mama. A continuación se presentan dos casos con el objetivo de describir un tipo de carcinoma de mama de presentación poco frecuente con evolución clínica no común. El primero de una mujer de 34 años y el segundo de una mujer de 44 años, ambas con diagnóstico confirmado de CAQ de mama. El CAQ de mama es de predominio en el sexo femenino en la etapa postmenopáusica. Sin embargo, ambas pacientes presentaron el CAQ en edad fértil. Generalmente posee un pronóstico favorable con rara diseminación, a diferencia de estos dos casos presentados donde se observa varias metástasis a distancia y un curso muy agresivo de la enfermedad.

\section{PALABRAS CLAVES: Cáncer de mama, carcinoma adenoideo quístico, cilindroma, adenocarcinoma quístico}

\section{SUMMARY}

Adenoid cystic carcinoma (ACC) of the breast is a rare neoplasm and accounts for $0.1 \%$ to $0.4 \%$ of all breast carcinomas. This is a description of two clinical cases that describe a rare presentation with an uncommon clinical course. The patients are 34 and 44 year old women, both with a confirmed diagnosis of ACC of the breast. The ACC of the breast predominantly grows in postmenopausal women. However, both patients are still fertile. Commonly it has a favorable prognosis and a rare presentation of metastasis. Nevertheless, in both cases, distant dissemination and an aggressive course of the disease was observed.

\section{KEY WORDS: Breast cancer, adenoid cystic carcinoma, cilindroma, cystic adenocarcinoma}

\section{INTRODUCCIÓN}

El carcinoma adenoideo quístico (CAQ) de mama es un cáncer poco frecuente y representa entre el $0,1 \%$ y el $0,4 \%$ de todos los carcinomas de mama (1). Esta enfermedad fue reportada por primera vez en 1946 por Foote y Steward (2).

También se le conoce como cilindroma, adenocarcinoma quístico, entre otros. Su localización más frecuente es en las glándulas salivales, pero puede aparecer en otras zonas como en la glándula lagrimal, mama, árbol traqueobronquial, laringe, cuello uterino, glándulas de Bartholino y próstata (3). En estas sus características histopatológicas son similares, pero la evolución y pronóstico difieren (3). 
Es más frecuente en mujeres post menopaúsicas con presentación de masa palpable única, unilateral e indolora. Tiene un pronóstico favorable por tener crecimiento lento (1). La afectación axilar local y metástasis a distancias son raras (4). El diagnóstico definitivo es anátomo-patológico y el tratamiento es la cirugía conservadora (4).

El objetivo de esta comunicación es presentar 2 casos clínicos, el diagnóstico y tratamiento de esta infrecuente neoplasia mamaria en el Hospital Nacional Edgardo Rebagliati Martins (HNERM).

\section{Caso clínico 1}

Paciente mujer de 34 años que refiere la presencia de una masa en la mama izquierda desde hace 4 años. Niega antecedentes quirúrgicos. Entre sus antecedentes ginecológicos, refiere menarquia a los 11 años, fecha de su última regla fue dos semanas antes de la primera consulta, 5 gestaciones, 3 partos y 2 abortos de causa no especificada. Su primer parto fue a los 24 años, y como método anticonceptivo utiliza un dispositivo intrauterino desde hace 5 años. Niega hábitos nocivos y otras patologías.

En la primera consulta, se encuentra una tumoración de $10 \times 10 \mathrm{~cm}$ que ocupa toda la mama izquierda, consistencia dura y leve dolor a la palpación. Además, se encontró un ganglio de $2 \mathrm{~cm}$ en la región axilar izquierda. No hubo hallazgos significativos en la mama derecha y en el resto del examen físico.

Se realizó una biopsia el mismo año consistente con un carcinoma metaplásico de mama con necrosis y fondo mixoide. En la macroscopía se evidencia un fragmento de tejido de 2,5 x 1,5 x 1 $\mathrm{cm}$, color amarillo blanquecino, superficie regular y consistencia elástica. Al corte, presenta superficie blanquecina con áreas amarillentas. La inmunohistoquímica muestra receptores estrogénicos negativos, receptores de progesterona positivos débilmente $(+/+++)$ en $80 \%$ de células y un C-ERB-2 negativo. El reporte anátomo-patológico establece una lesión consistente con un carcinoma adenoideo quístico (Figura 1). El reporte tomográfico, realizado 6 meses después del diagnóstico definitivo, muestra una lesión en mama izquierda de $5 \times 4 \mathrm{~cm}$ sin compromiso de arcos costales. La gammagrafía ósea no presentó hallazgos significativos. Por lo tanto, el diagnóstico definitivo es CAQ T3N1M0 en EC IIIB.

La paciente recibe quimioterapia con Cisplatino-5 Fluorouracilo (ciclos y dosis no registrados). Se realiza una mastectomía radical modificada de tipo Madden con resección de la pared del músculo pectoral mayor. Un año después, la tomografía muestra lesión expansiva blanda de unión costoesternal media izquierda de $3 \mathrm{~mm}$ (probable metástasis), pulmones normales y sin adenopatías.
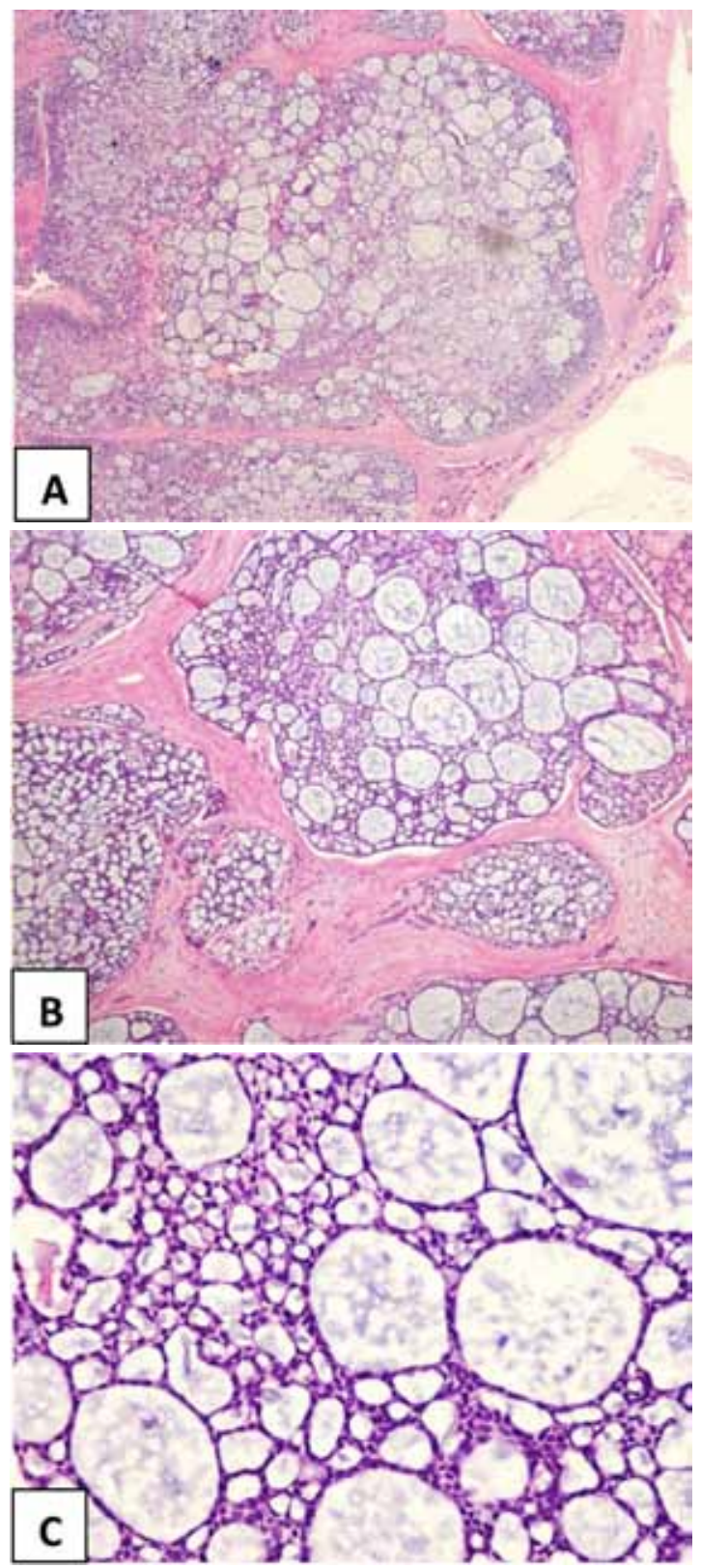

Figura 1. Caso 1. Características histopatológicas del carcinoma adenoideo quístico: A. Tinción HE 40x B. Tinción HE 100x C. Tinción HE 400x.

La paciente recibe radioterapia esternal de 3000 cG4 (10 sesiones). Un año después, la tomografía evidencia un micronódulo perihiliar derecho de 5 $\mathrm{mm}$, resto de los pulmones normales, presencia de masa con densidad de partes blandas paraesternal izquierda de $35 \times 28 \mathrm{~mm}$ con osteolisis esternal y 
parcial necrosis central en relación con implante tumoral por debajo del manubrio esternal. Además, la gammagrafía ósea reporta en el tercio superior del esternón un área con mayor concentración de contraste, lesión sospechosa de metástasis ósea. Aproximadamente 4 meses después, el reporte tomográfico informa una lesión focal blanda expansiva de $3 \mathrm{~cm}$ en región condroesternal izquierda sugerente de metástasis, múltiples nódulos metastásicos en ambos pulmones, escasas adenomegalias en mediastino medio pretraqueal hasta $1 \mathrm{~cm}$. Asimismo, la gammagrafía ósea mostro metástasis pulmonares múltiples de tipo nodular menos de $18 \mathrm{~mm}$ en tórax. La paciente continúo recibiendo quimioterapia y radioterapia en mama izquierda. El último control de la paciente fue en el año 2008 por el servicio de oncología del HNERM, según los registros de la institución, la paciente ha fallecido de causa no especificada.

\section{Caso clínico 2}

Paciente mujer de 44 años con tiempo de enfermedad de 3 años, refiere presencia de tumoración indolora de crecimiento progresivo en mama derecha desde el año 2001. Dos años después, se realiza ecografía de mama derecha con características benignas, y no recibe tratamiento. Un año después, la tumoración aumenta de tamaño, por lo es referida al HNERM desde el hospital de Puno. En la primera consulta se palpa tumoración en mama derecha de $7,5 \times 7,0 \mathrm{~cm}$, consistencia pétrea y adherido a planos profundos con compromiso cutáneo, mama izquierda sin alteraciones. Entre sus antecedentes ginecológicos, destaca menarquia a los 10 años, 2 gestaciones y 1 aborto de causa no especificada. Primera gestación a los 35 años, niega uso de métodos anticonceptivos, histerectomizada por miomas hace 4 años, sin terapia de reemplazo hormonal. Niega enfermedades previas, hábitos nocivos y antecedentes familiares desfavorables.

Se efectúa biopsia que fue compatible con carcinoma intraductal de mama de bajo grado con receptores estrogénicos negativos, receptores progesterona positivos $+/+++$ en $5 \%$ de las células y HER2 negativo. A finales del año 2005, la anatomía patológica es compatible con carcinoma ductal infiltrante de células pequeñas con componente de CAQ (Figura 2), receptores estrogénicos positivos $+/+++$, receptores de progesterona $++/+++$ en el $80 \%$ de sus células. Macroscópicamente se obtuvo una pieza tumoral de $3 \times 2 \times 2 \mathrm{~cm}$, color blanquecino, aumento de consistencia, sin compromiso de pezón ni de grupos ganglionares $(0 / 26)$. Por lo tanto, el diagnóstico definitivo es CAQ T4b NO MO EC IIIB. Se realizó mastectomía modificada tipo Madden, quimioterapia neo adyuvante con cisplatino y placlitaxel por 6 ciclos (dosis no registrada), asociada a radioterapia (10 sesiones).
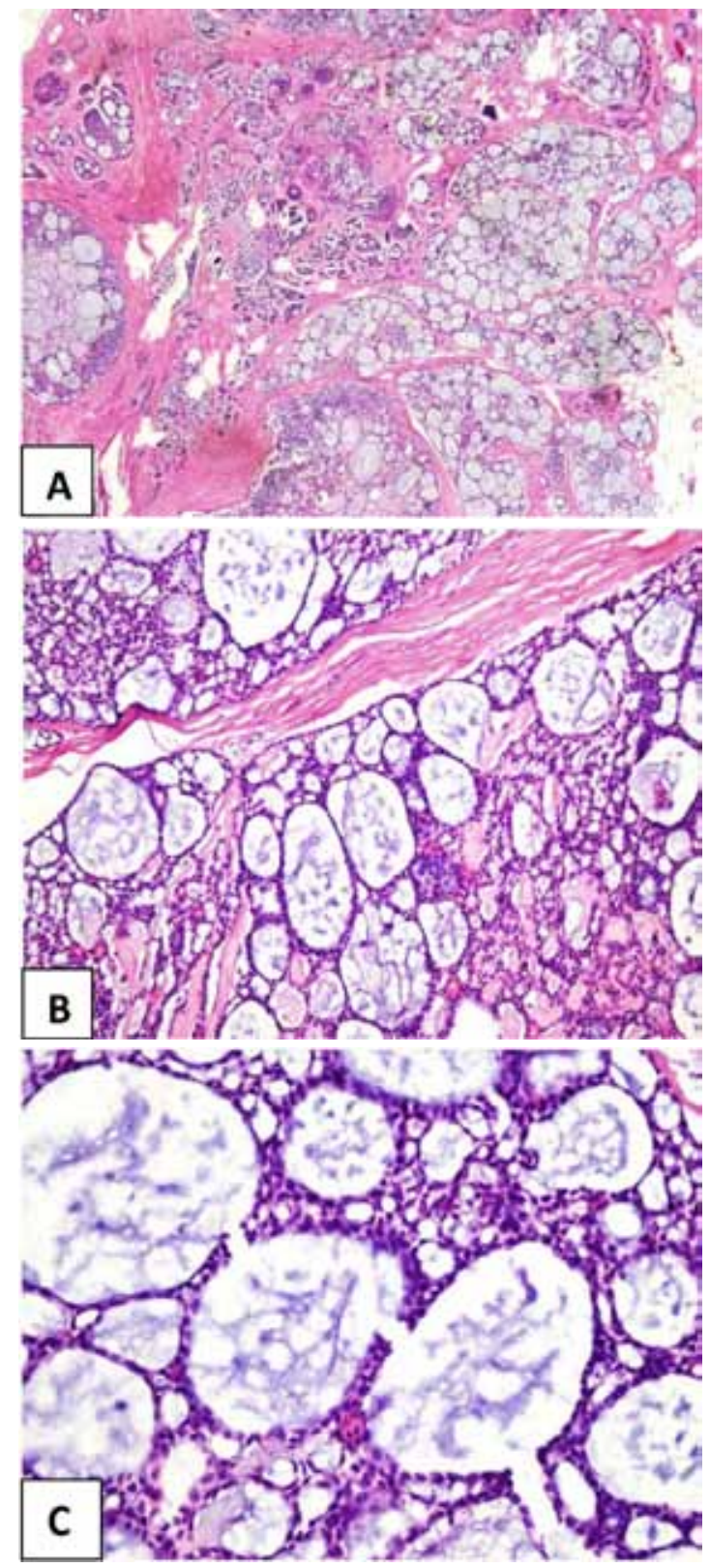

Figura 2. Caso 2. Características histopatológicas del carcinoma adenoideo quístico. A. Tinción HE 40x B. Tinción He 100x C. Tinción HE 400x.

Seis años después se evidencia en la TAC una formación hipodensa irregular comprometiendo el lóbulo izquierdo de la glándula tiroides, múltiples adenopatías de $50 \mathrm{~mm}$ en hilio pulmonar derecho, nódulos de aspecto metastásico en región parahiliar 
izquierda y lóbulo superior derecho en segmento anterior e inferior. Además, se encontró un nódulo sólido de aspecto metastásico en glándula suprarrenal izquierda y una masa sólida de aspecto metastásico en ambos riñones. Dos años después, se realiza biopsia del pulpejo del cuarto dedo de la mano izquierda compatible con metástasis de origen mamario. Ese mismo año, se trató con inhibidores de la aromatasa (sin especificación). Actualmente, según los registros de la institución, la paciente ha fallecido sin especificación de causa $y$ año de deceso.

\section{DISCUSIÓN}

Es importante resaltar la edad de ambas pacientes, una de ellas era aún fértil, de 34 años de edad y la otra paciente tenía 41 años al inicio de la enfermedad. La edad diagnóstica del CAQ de mama es de 19 a los 97 años, con predominio en la quinta y sexta década de vida $(3,4)$. Es común su presentación en postmenopáusicas, siendo la aparición en mujeres jóvenes y varones fenómenos muy raros. Al examen físico, lo más frecuente es la presencia de una masa blanda e indolora (3).

Una de las pacientes inicia su enfermedad con un cáncer ductal infiltrativo que después muestra en el análisis histopatológico tejido de un CAQ. Histológicamente se caracteriza por un patrón bifásico de glándulas verdaderas bordeadas por células epiteliales y pequeñas células mioepiteliales basaloides alrededor de espacios pseudoquísticos (6). La metástasis ganglionar en ambas pacientes es una presentación rara de la enfermedad (5). Hay pocos casos reportados de pacientes con ambos tipos de cáncer en la misma lesión, estas suelen presentar afectación ganglionar, metástasis óseas y pulmonares entre otras. A diferencia del CAQ puro estos tumores mostraron un comportamiento agresivo (7).

Ambas pacientes recibieron quimioterapia y radioterapia como parte del tratamiento. Se recomienda la extirpación total de este tumor, sin la necesidad de resección ganglionar (4). En relación con la quimioterapia en la enfermedad a distancia, no hay suficiente evidencia por su escasa casuística. Sin embargo, se ha visto el uso frecuente de cisplatino, adriamicina, docetaxel y ciclofosfami$\mathrm{da}$, entre otros en el tratamiento neoadyuvante y coadyuvante $(4,6)$.

Las pacientes presentaron recurrencia posterior a la mastectomía, una de ellas presentó metástasis pulmonar y la otra metástasis ósea, pulmonar, tiroidea, renal y suprarrenal. El rango de recurrencia de este tumor esta entre el $6 \%$ al $37 \%$ luego de una resección local y la recurrencia después de una mastectomía es extremadamente rara (4). La supervivencia a los 5 años es $85 \%$ incluso tras metástasis (3). Es importante recalcar la importancia de reportar presentaciones atípicas del CAQ, ya que al tener un buen pronóstico, ambos casos presentaron un curso agresivo y poco favorable. Ambas pacientes a la fecha se encuentran fallecidas. Estas presentaciones de curso agresivo ayudan a ampliar el desenlace esperado al diagnosticar una patología de buen pronóstico. Tomando en cuenta la mortalidad que puede causar, se podría considerar desde un estadio clínico más temprano un tratamiento más agresivo.

\section{REFERENCIAS}

1. De Luis E, Apesteguía L, Noguera JJ, Pina L, Martinez-Requeira F, Miguel C, Saenz J. [Adenoid cystic carcinoma of the breast]. Radiologia 2006;48(4):23540.

2. Foote FW Jr, Stewart FW. A histologic classification of carcinoma of the breast. Surgery. 1946;19:74-99.

3. Pérez-López E, García-Gómez J, García-Mata J, Salgado-Fernández M, Fírvida-Pérez J. Carcinoma adenoide quístico de mama: a propósito de un caso. Prog Obstet Ginecol 2006;49(2):85-8.

4. Pérez JA, Poblete MT, Deichler F, Fuentes M. Carcinoma adenoideo quístico mamario. Reporte de un caso. Cuad Cir 2006;20:70-4.

5. Arpino G, Clark GM, Mohsin S, Bardou VJ, Elledge RM. Adenoid cystic carcinoma of the breast molecular markers, treatment, and clinical outcome. Cancer 2002;94(8):2119-27.

6. Boujelbene N, Khabir A, Boujelbene N, Jeanneret Sozzi W, Mirimanoff RO, Khanfir K. Clinical review -- breast adenoid cystic carcinoma. Breast 2012;21(2):124-7.

7. Righi A, Lenzi M, Morandi L, Flamminio F, De Biase $D$, Farnedi A, Foschini M. Adenoid cystic carcinoma of the breast associated with invasive duct carcinoma: a case report. Int J Surg Pathol 2011;19(2):230-4. 